\title{
Tuning the Optical Properties of Quantum Dots to Increase the Efficiency of Solar Cells
}

\author{
Arnav Ghatiwala \\ Independent \\ Jaipur, India
}

\begin{abstract}
Quantum Dot Solar Cells (QDSC) are a promising class of third-generation solar cells that are being developed to deliver pathbreaking efficiencies as a result of the unique optical properties of quantum dots, and this paper aims to increase their currently dismaying efficiency. Based on the size-based tunability of quantum dot band gaps, a model is developed that relies on absorbing a larger span of energies for photons to efficiently convert them to excitons. By considering a set of binary compounds used as semiconductor materials in quantum dots and processing them through mathematical equations derived from Schrödinger's equation for an electron in an infinite potential well, a final group of candidates in a range of sizes are selected based on their wavelength absorption ranges and coverage of the solar spectrum. These quantum dot candidates are then finalized considering material characterizations and are arranged in a photovoltaic cell to generate maximum efficiency. A layer-by-layer process is discussed for deposition, while a method of effective size distribution is proposed. An analysis of energy losses including electron recombination and surface defects are further discussed, and solutions are proposed. The end product is a framework for highly efficient quantum dot structures within a QDSC that can be employed commercially.
\end{abstract}

Keywords - Nanotechnology; quantum dots; photovoltaics; band gap; excitons; power conversion efficiency

\section{INTRODUCTION}

With energy needs growing worldwide and a decrease in the amount of fossil fuel, there has been a massive increase in the importance of alternative and renewable energy sources, including solar, wind, tidal, geothermal, etc. While solar energy presents as the most feasible option, its poor efficiency in comparison to other sources has led to it meeting less than $2 \%$ of the world's total energy needs in 2019[1]. Continuous research and innovation have increased the efficiency of solar photovoltaic panels over the years, making it less expensive from a consumer perspective. However, the efficiency of most solar panels still ranges from $15-17 \%$ [2], which is a meagre value in the energy industry. This is mainly because in conventional single-junction solar cells (also known as first-generation solar cells) a fixed amount of energy is harvested from a solar photon, even though the solar spectrum includes photons with energies ranging from $0.4 \mathrm{eV}$ to $4.0 \mathrm{eV}$. The fixed amount of energy converted to electrical power is determined by the band gap of the semiconductor. If the energy of the photon is lower than the band-gap energy, the photon is not absorbed. Moreover, if a photon has energy greater than the band-gap energy, a high amount of this energy is lost via thermalization as phonons are emitted.
Figure 1 shows the portion of the solar spectrum that can be absorbed by an ideal traditional solar cell and the ensuing losses. Since at least $33 \%$ of available energy is lost due to thermalization and $19 \%$ is not absorbed because it has a greater wavelength than the solar cell's band gap, there is a possibility to increase the power conversion efficiency (PCE) of the photovoltaic panel by transforming unused or lost energy into electrical power. This has been recognized by researchers worldwide, who are trying to develop novel methods to achieve higher PCE values. Many of the recent methods have been clubbed under the umbrella of thirdgeneration photovoltaics (PVs), and it is interesting to explore these.

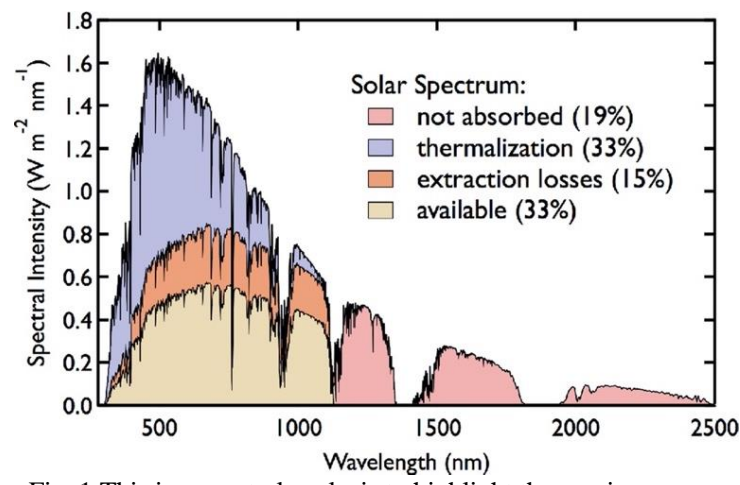

Fig. 1 This is a spectral analysis to highlight the maximum energy available from a single-junction solar cell with a semiconductor with a band gap of 1.1eV operating under the AM1.5 (Air Mass 1.5) global spectrum. This analysis was first presented by Shockley and Queisser in 1961 and the minimal losses in the diagram represent the Shockley-Queisser limit. [3]

\section{A. Reviewing Third-Generation PVs}

Current first-generation and second-generation solar cells only approach the Shockley-Queisser(SQ) limit of converting $33 \%$ of the total energy from sunlight to electrical energy; for example, the record for c-Si based solar cells is currently at $25 \%$ while for GaAs the record is $28.3 \%$ [4]. However, there have been many attempts to bypass this limit, and these can be grouped under the umbrella of third-generation solar cells. Third-generation solar cells can have a higher limiting conversion efficiency by bypassing one of the assumptions of the SQ analysis and recovering either some of the energy lost via thermalization or providing pathways to harvest those photons not absorbed in a standard solar cell[5]. In essence, they aim to increase the efficiency of solar cells while still using thin-film second-generation deposition methods. The concept is to do this with a minimal increase in areal costs and hence reduce the cost per Watt peak, a metric commonly used in the PV industry. The aim is to reduce costs way below the $\$ 1 / \mathrm{W}$ level of the second-generation QDs, down to 
$\$ 0.50 / \mathrm{W}$, or even as low as $\$ 0.20 / \mathrm{W}$. By having higher efficiency with similar deposition methods, more power can be generated by the same area of a solar cell, and thus users need to buy a smaller number of solar panels to satisfy the same energy needs, reducing costs. The efficiency and cost considerations of the three generations of solar cells can be understood from Figure 2.

Tinted areas:

$67-87 \%$ representing thermodynamic limit

$31-41 \%$ representing single bandgap limit

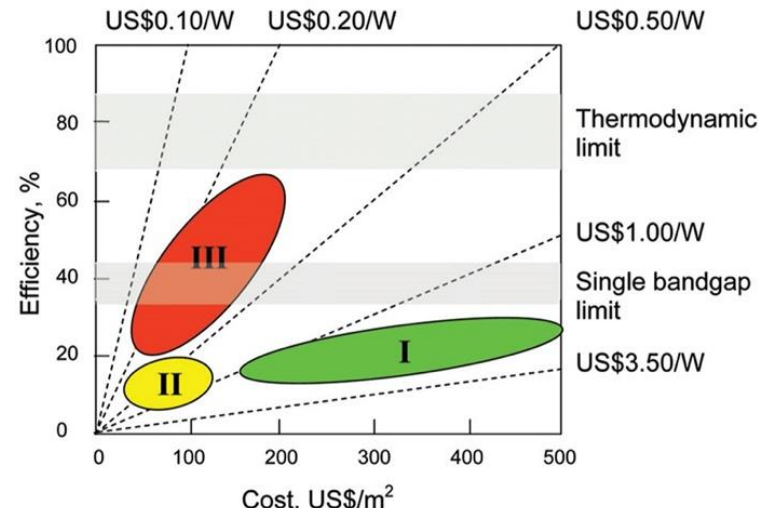

Fig. 2 The efficiency and cost projections for first, second, and thirdgeneration PVs[6]

There are several third-generation approaches including multi-junction solar cells, intermediate band solar cells, multiple exciton generation (MEG), up-and-down conversion, and hot carrier cells. Concepts like intermediate band solar cells involve introducing an energy level within the band gap to absorb photons in parallel with the normal operation of the cell. Moreover, up conversion involves absorbing two photons and producing one higher energy photon for absorption, while down conversion involves absorbing a single photon to output two photons with lower energies. However, we will be focusing on quantum dot solar cells (QDSCs) which hold great promise for the highest PCEs. Furthermore, research into the use of quantum dots in PVs can also lead to their employment as the major components in the other types of third-generation solar cells.

\section{B. Quantum Dot Fundamentals}

Quantum Dot Solar Cells were first proposed in 1990 by Barnham and Duggan as a substitute for regular solar cells [7]. Quantum dots (QDs) are a special form of semiconductors, which are three-dimensionally confined, taking the form of nanocrystals, and are thus useful due to the unique properties that arise as a result of their size regime. Their semiconductor composition means that electrons in QDs bridge the intrinsic band gap when excited by photons in the incident light, much like bulk semiconductors due to the photoelectric effect. However, while bulk semiconductors are characterized by continuous valence and conduction bands, quantum dots are too sparse to create this structure because they are formed of very few atoms (being less than 100 in diameter). Therefore, they create a rarified electronic structure that compares to the discrete energy states in single atoms. The band gap of a QD then depends on its size, as the smaller the quantum dot, the more the energy levels space out, causing the band gap to increase. We then arrive at the attractive property of quantum dots that make them useful in solar cells; unlike single atoms, the reliance of the band gap on the size of the nanocrystal allows researchers to tune its band gap energy by adjusting its size. In essence, we can create quantum dots with arbitrary band gap energies to cover a greater span of the solar spectrum.

This tunability of the band gap energies of QDs can be attributed to a special phenomenon that takes place in these nanoparticles: quantum confinement. In a quantum dot, an electron is excited to the conduction band from the valence band as a photon strikes the dot with sufficient energy. In the place of that electron, there is now a positive hole, and this electron-hole pair which is generated is called an 'exciton'. Uniquely, in QDs, the exciton Bohr radius (the distance between the electron and the hole) is the same order as or smaller than the size of the quantum dot itself. As the exciton is squeezed into the nanocrystal, confinement energy is produced, and the pronounced confinement causes discrete, quantized energy levels which are spaced out. This electronic structure resembles an atom, which is why quantum dots are even referred to as 'artificial atoms'. Furthermore, as the quantum confinement causes the energy levels to space out, the band gap energy increases, too.

Though band gaps can be tuned based on size, it is important to understand other optical properties of QDs. It has been proven that the quantum confinement effect in QDs enhances multi exciton generation, where a single photon can lead to the formation of multiple excitons when the photon energy is greater than the band-gap energy. Even though the focus of this paper will be to explore the size-dependent variation in the band gap and optimizing this aspect, it is also crucial to highlight the other properties of QDs that can make them excellent choices for use in solar cells. Thus, incorporating quantum dots in solar cell structures has immense potential to minimize losses from thermalization and unabsorbed photons.

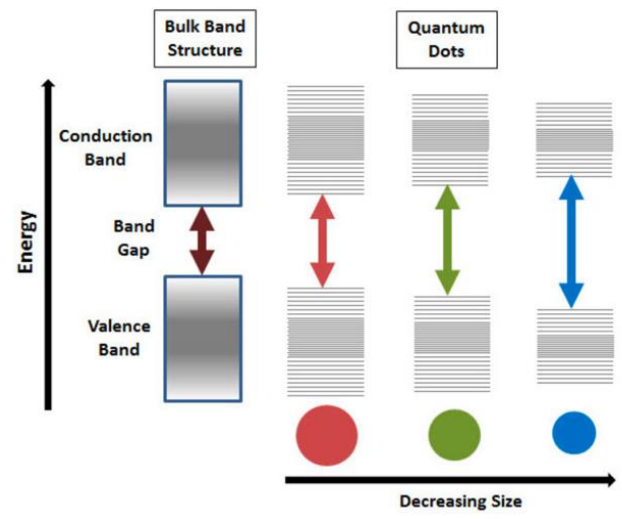

Fig. 3 The electronic structure of the QD varies with their size [8]

\section{Scope of QDSCs}

Before delving deeper into the optical properties of QDs and discussing their incorporation in solar cells, it is integral to understand the value they add to solar panels. Initial interest in quantum dots by photovoltaic researchers was due to their high absorption, which could reduce absorber 
material requirements. Since they have higher orders of absorption than traditional thin films and silicon, the QD based solar devices can generate higher photocurrents. Today, the key factor behind the introduction of quantum dots in solar panels is clear: the possibility to increase their efficiency. This is true due to the possibility of minimization in energy losses, as described earlier. Where the absorption spectra for QDs shifts with their size, a correct combination of sizes and materials of QDs in the cell could lead to much greater coverage of the incident solar spectrum, including covering the $50 \%$ of the spectrum which lies in the infra-red or near infra-red region and is not absorbed by silicon. Finding this correct combination becomes the aim of this paper, but it is integral to understand the benefits of this combination before moving ahead. Quantum dot solar cells can make solar energy more cost-competitive with other sources of energy, resulting from their potential to have $65 \%$ efficiency [9]. Having a low power to weight ratio, these cells can result in mass and area savings with flexibility leading to miniaturization. Furthermore, even the process of synthesizing QDs and depositing them on the electrode in the solar cell is relatively inexpensive. The need for alternative energy has been established beforehand, and QDSCs can make solar energy a potent substitute for energy from fossil fuels.

However, there are multiple reasons why QDSCs have NOT already become commercially available worldwide and used on a larger scale. Despite numerous advantages, the highest efficiency for QDSCs in laboratories is 4-5\%. This is because QDSCs are an emerging field, and it will take decades of research to optimize these cells' material and device characterizations. Today, deficiencies in their structure relating to the materials and other specifications cause the low efficiency. While the electrolyte used for dye-sensitized solar cells proved to be corrosive to QDs, researchers have come up with an alternative - polysulfide liquid electrolyte. However, this new alternative has high catalytic activity with the counter electrode, increasing internal series resistance. Another problem lies in the deposition methods used, where QDs can be fabricated and deposited simultaneously or separately. In situ, methods like successive ionic layered absorption and reaction (SILAR), chemical bath deposition (CBD) and electrodeposition let the QDs grow directly onto the porous substrates. Ex-situ QDs are fabricated separately and deposited onto the substrate with a linker [10]. Providing better electric conveyance and increased absorption for quantum dots, in situ methods are generally favored. However, such techniques lead to a low percentage of the thin film surface being covered by QDs. If we take the case of the deposition of cadmium sulfide on a thin porous titanium dioxide film, only about $20 \%$ of the surface is covered. These low concentrations result in lower photocurrents and higher electron recombination with the electrolyte, leading to higher internal shunt resistance and low fill factors. In essence, all this means that the efficiency of the cell decreases. Apart from this, quantum dot solar cells also present a health hazard since the most commonly used materials are highly toxic. Cadmium Selenide, which is most commonly used in quantum dot cores for solar cells, is composed of cytotoxic ions.
While extensive research continues in the field of quantum dots, their possibility for unseen levels of solar cell efficiency is currently inhibited by material and design problems as described above. This research work has acknowledged the engineering problems that may be encountered in the other parts of QDSC besides the quantum dots or the problems relating to quantum dot synthesis. Thus, I will set out to create a model to absorb a much larger span of the solar spectrum through our cell, simultaneously keeping the engineering limitations in mind.

\section{TOWARDS BETTER QDSCS}

Increasing the efficiency in quantum dot solar cells can be achieved through first absorbing as large a part of the solar spectrum as possible to convert solar energy to electric energy. By incorporating multiple QD materials in our solar cell, each in different sizes of nanocrystals, we could create a larger number of excitons, which could then result in more electrons being extracted to become a part of the circuit and giving greater power efficiency. I thus set out to create a model of a QDSC by analyzing QDs of different semiconductor materials, calculating their ideal absorption ranges through our mathematical equations, and then shortlisting the ideal candidates for the cell with the suggested range. While we have already introduced a solar spectrum in Figure 1, highlighting how merely a theoretical maximum of $33 \%$ of the available energy can be harnessed by a singlejunction silicon semiconductor solar cell, this model now aims to harness more of the available energy by employing quantum dots in our cell. Initially, we will attempt to cover the maximum wavelengths of the spectrum by employing multiple materials. Thus, we will use an AM1.5 (Air Mass 1.5) solar spectrum, where AM1.5 is used to represent the overall yearly average for mid-latitudes. In the solar panel industry, this air mass index is considered ideal for considering the spectrum, as the specific value of 1.5 has been selected in the 1970s for standardization purposes, based on an analysis of solar irradiance data in the conterminous United States [11].

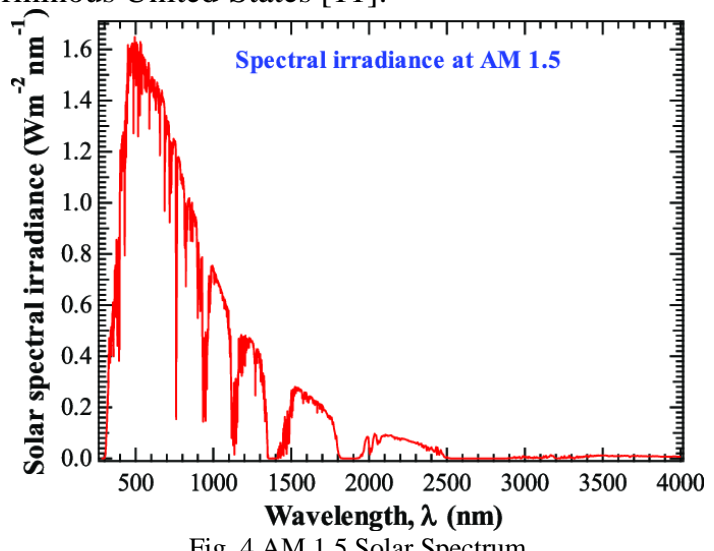

The analysis of multiple semiconductor materials that are used as quantum dots can allow us to determine their ideal absorption ranges based on the size they can be synthesized in. Moreover, this should allow the selection of ideal candidates to be deposited on the solar cell and the size ranges required. It will also become important to analyze 
energy losses as solar energy is converted to electrical energy, thus creating the basis for a quantum dot solar cell that can be employed for increased efficiency. This methodology is novel and researching and modelling a multi-material solar cell based on covering various wavelengths could prove to be highly useful.

\section{A. Mathematical Modelling}

An integral part of our model involves deriving the ideal wavelength absorption range for QDs of different materials based on their size range. It was earlier established that the band gap energy of a QD can be expressed as a function of its radius. Thus, the required energy for an incoming photon and even its wavelength can be expressed in terms of the radius of the quantum dot. This can be done by bringing together the different energies involved in a semiconductor nanocrystal apart from the band gap energy of the bulk semiconductor, i.e. the confinement energy and the energy associated with the coulombic attraction. The quantum confinement in QDs can be explained by the infinite potential quantum well model, where the quantum dot is now a quantum box. The quantum regime is entered as the size of the semiconductor crystal decreases, where the electronic properties are strongly dependent on the electron and hole in a confined space. Deriving from Schrodinger's equation for an electron in an infinite potential well [12], I thus arrived at the confinement energy for the quantum dot, as shown below in Equation 1:

$$
E_{\text {confinement }}=\frac{\hbar^{2} \pi^{2}}{2 a^{2}}\left(\frac{1}{m_{e}}+\frac{1}{m_{h}}\right)=\frac{\hbar^{2} \pi^{2}}{2 \mu a^{2}}
$$

Here, $\mu$ is the reduced mass of the exciton system, a is the radius of the quantum dot, $m_{e}$ is the effective mass of the electron, and $m_{h}$ is the effective mass of the hole.

It is also interesting to note how the confinement regime the quantum dot is linked to the result of changing size on the band gap. The confinement regime is based on the comparison between the exciton Bohr radius $\left(a_{b}\right)$ and the size of the quantum dot. The exciton Bohr radius can be calculated for a material using Equation 2. Coming to the confinement regimes, if the exciton Bohr radius is on the same order as the radius of the quantum dot, it is said to be in the 'weak confinement regime'. Similarly, if the exciton Bohr radius is larger than the radius of the $\mathrm{QD}$, the dot is now placed in the intriguing 'strong confinement regime' where confinement effects dominate significantly. For the band gap to be easily variable as a function of size, the quantum dots must not have a size larger than their exciton radius to achieve the band gap tunability that we require for my model.

$$
a_{b}=a_{0} \varepsilon_{r}(2)
$$

Here, $\mathrm{a}_{0}$ is the Bohr radius (approximately $0.53 \AA$ ) and $\varepsilon_{\mathrm{r}}$ is the dielectric constant of the semiconductor.

Furthermore, we observe another energy associated with the electrostatic attraction in the exciton between the negative electron and the positive hole, as shown in Equation 3:

$$
E_{\text {exciton }}=-\frac{1}{\varepsilon_{r}^{2}} \frac{\mu}{m_{e}} R_{y}
$$

Here, $\mathrm{R}_{\mathrm{y}}$ is the Rydberg energy, which is approximately equal to $13.6 \mathrm{eV}$. From here, we can go on to calculate the exact energy needed by a photon to excite an electron from the valence band to the conduction band in a quantum dot. This photon will need to provide energy greater than the band gap of the bulk semiconductor, as there are now the additional associated confinement and exciton energies in a quantum dot. By analyzing the likelihood of a change in the photochemical redox potential of a carrier as a result of changing QD size, we were able to model the elementary quantum mechanics of a nanocrystal by using effective mass approximation (EMA), an electrostatic potential for dielectric polarization, and penetration of the carrier outside the nanocrystal in a case of small effective mass. Louis Brus [13] had developed a quantum model for spherical quantum dots, but we would be altering the expression concerning the electrostatic attraction to better suit the needs of this paper. Therefore, we have modelled the energy needed by a photon in Equation 4, which is essentially the first exciton energy:

$$
\begin{aligned}
E_{\text {photon }}=\frac{h c}{\lambda} & =E_{\text {bandgap }}^{\text {bulk }}+E_{\text {confinement }}+E_{\text {exciton }} \\
& =E_{\text {bandgap }}{ }^{\text {bulk }}+\frac{\hbar^{2} \pi^{2}}{2 \mu a^{2}}-\frac{1}{\varepsilon_{r}{ }^{2}} \frac{\mu}{m_{e}} R_{y} \text { (4) }
\end{aligned}
$$

This equation is representative of how altering a, the radius of the quantum dot, can affect the exact energy needed to excite an electron, i.e. the first exciton energy or the band gap energy of the quantum dot. Further simplification and modification of this equation allow us to represent the wavelength of light needed to excite an electron just enough to cover the band gap, as a function of the QD radius. This is shown in Equation 5:

$$
\lambda=\frac{h c}{E_{\text {bandgap }}{ }^{\text {bulk }}+\frac{0.376}{\beta a^{2}}-\frac{R_{y} \mu}{\varepsilon_{r}{ }^{2} m_{e}}}
$$

We have modified the equation to be represented in terms of wavelength. Furthermore, we have simplified the term for the confinement energy by replacing $h_{\text {bar }}$ with $h$, multiplying the numerator and denominator with $\mathrm{c}^{2}$, and then replacing constants with their known values. $\beta$ represents the reduced mass of the exciton system, divided by the mass of an electron. Therefore, this equation is integral to understanding the specific wavelength of light that quantum dots with particular materials and radii can absorb. When a quantum dot absorbs the photon and the electron is excited, it is then extracted from the dot to flow in the circuit, forming the current. In essence, this equation enables us to understand the exact wavelength needed to create an exciton with minimal losses for a quantum dot with a particular size. Figure 5 illustrates how this wavelength varies for CdTe quantum dots with their size. While the extraction of the electron faces other difficulties, my aim here would be to create maximum excitons that can then be extracted to give a greater power output. 


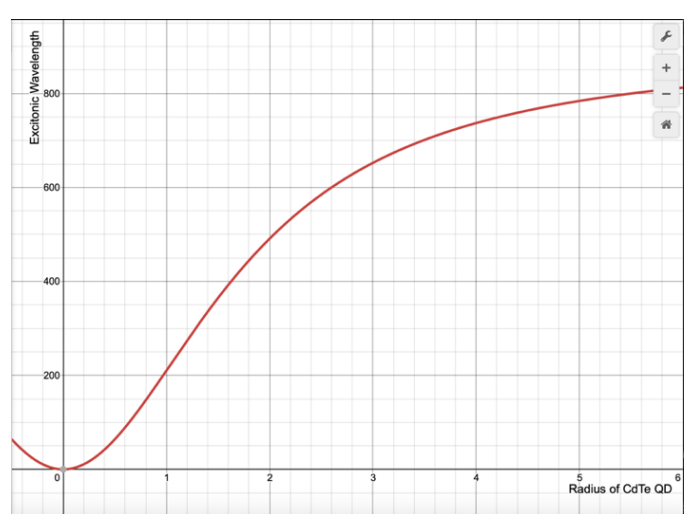

Fig -5: The excitonic wavelength (in nanometres) of CdTe QDs represented as a function of their radius (in nanometres).

\section{B. Selection and Analysis of Materials}

To select and place quantum dots of different materials and sizes for solar cells, the initial step was to create a list of semiconductor substances that could be used in the form of quantum dots. The search was focused on nanocrystals formed by compounds belonging to the periodic groups of II-VI, III-V, or IV-VI materials, which have been conventionally used as the constituent material in quantum dots due to their excellent semiconducting properties. Furthermore, it was also integral to consider the durability of these materials as quantum dots, since a major obstacle for QDSCs is the high degradation of quantum dots. While halide perovskite QDs are being considered as the future of PVs [14], there is still much research to be conducted before such materials can be incorporated into solar cells on a broader basis. Silicon, despite being the most widely used semiconductor in traditional solar cells, is not as advanced for quantum dots [15] compared to the materials we selected. We looked for materials which had been extensively researched upon and tested as QDs in solar cells while showing effectiveness in multiple aspects relating to PVs, such as cadmium selenide and cadmium telluride. $\mathrm{CdSe}$ and CdTe, representing the II-VI periodic groups, are one of the most highly represented materials in QDSCs. We also selected a few materials which have not been as widely employed as nanocrystals in PV applications as the rest but could prove to be crucial as a result of their extreme band gap energies, allowing them to absorb extreme wavelengths in the solar spectrum. Aluminum nitride, with a band gap of 6.015 for the bulk semiconductor, showed promise to absorb in the ultraviolet range, but it has relatively less information available regarding its performance in QDSCs. Based on such reasoning, we were able to shortlist 16 candidates and procured the appropriate values associated with them that were integral to our calculations, as shown in Table 1.

TABLE 1

Parameters utilized for ideal wavelength calculations using the mathematical model [16][17][18][19][20][21][22]

\begin{tabular}{|l|l|l|l|l|}
\hline $\begin{array}{l}\text { Material } \\
\mathrm{s}\end{array}$ & $\begin{array}{l}\text { Band gap } \\
\text { energy } \\
\text { (bulk) / eV }\end{array}$ & $\begin{array}{l}\text { Effective } \\
\text { mass of } \\
\text { electron, } \mathrm{m}_{\mathrm{e}}\end{array}$ & $\begin{array}{l}\text { Effective } \\
\text { mass of hole, } \\
\mathrm{m}_{\mathrm{h}}\end{array}$ & $\begin{array}{l}\text { Dieletric } \\
\text { constant, } \varepsilon \mathrm{r}\end{array}$ \\
\hline $\mathrm{CdS}$ & 2.53 & 0.20 & 0.53 & 8.28 \\
\hline $\mathrm{CdSe}$ & 1.74 & 0.13 & 0.30 & 9.56 \\
\hline $\mathrm{CdTe}$ & 1.50 & 0.11 & 0.35 & 10.3 \\
\hline $\mathrm{PbS}$ & 0.37 & 0.085 & 0.085 & 161.0 \\
\hline
\end{tabular}

\begin{tabular}{|l|l|l|l|l|}
\hline $\mathrm{PbSe}$ & 0.26 & 0.07 & 0.06 & 227.0 \\
\hline $\mathrm{PbSe}$ & 0.29 & 0.24 & 0.30 & 450.0 \\
\hline $\mathrm{PbTe}$ & 0.36 & 0.028 & 0.33 & 15.2 \\
\hline $\mathrm{InAs}$ & 1.28 & 0.065 & 0.40 & 15.0 \\
\hline $\mathrm{InSb}$ & 0.17 & 0.013 & 0.18 & 17.9 \\
\hline $\mathrm{GaAs}$ & 1.43 & 0.067 & 0.45 & 12.9 \\
\hline $\mathrm{GaP}$ & 2.25 & 0.13 & 0.67 & 10.2 \\
\hline $\mathrm{GaSb}$ & 0.69 & 0.045 & 0.39 & 15.7 \\
\hline $\mathrm{GaN}$ & 3.40 & 0.20 & 0.80 & 8.90 \\
\hline $\mathrm{ZnS}$ & 3.54 & 0.28 & 0.50 & 8.00 \\
\hline $\mathrm{AlAs}$ & 2.21 & 0.15 & 0.75 & 8.50 \\
\hline $\mathrm{AlN}$ & 6.02 & 0.40 & 3.53 & 10.1 \\
\hline
\end{tabular}

The array of materials selected for analysis shows how it was crucial to have materials with diverse band gaps, dielectric constants, and effective masses. With the data having been collected, it was now time to process it through our Python 3 model to output the results in terms of wavelengths that we required. The model was specifically created and coded for this research project to access the values of the parameters that we collected, process the data, and output the wavelength ranges for all our materials. However, it was imperative to know the size ranges that QDs of different materials are commonly synthesized in since the radius of the quantum dot is a crucial variable in the equations. There has been ample experimental proof of the span of diameters/radii that have been created for the QDs we have selected. We also made sure to take the size data for a range that is commonly produced and not a one-off exception. This way, our cell would be easily implementable in reality instead of being constrained to a theoretical idea.

Our candidates broadly fit under a range of 2-10 nm (diameter), which is the characteristic size of QDs. For most QDs, a size larger than this would result in weaker quantum effects as the radius of the nanocrystal becomes larger than the exciton radius, falling in a weak confinement regime. Our first candidate was Cadmium Sulfide, or $\mathrm{CdS}$, which has commonly been used in QDSCs due to rapid advancements in the available knowledge of their synthesis and use as QDs. Synthesis methods that are capable of controlling the size of binary compound semiconductors have been developed and reported [23], especially in the case of II-VI compounds. Cadmium chalcogenide QDs can be prepared easily with a high level of control over size, morphology, and composition, making them preferable additions to the cell. We have taken the radius range for $\mathrm{CdS}$ QDs that is synthesized in a colloidal solution which can be deposited on the panel, being 1.0-4.5 $\mathrm{nm}$ [24]. The wavelengths to cover the band gap for the smallest and largest crystallite can then be calculated in the following manner using Equation 5: 


$$
\begin{aligned}
& \lambda_{\text {shortest }}=\frac{h c}{2.53+\frac{0.376}{0.145 \times 1.0^{2}}-\frac{13.6 \times 0.145}{8.28^{2} \times 0.20}}=249 \mathrm{~nm} \\
& \lambda_{\text {longest }}=\frac{h c}{2.53+\frac{0.376}{0.145 \times 4.5^{2}}-\frac{13.6 \times 0.145}{8.28^{2} \times 0.20}}=494 \mathrm{~nm}
\end{aligned}
$$

These values for the wavelengths were outputted by our Python model, allowing us to understand the wavelengths of light our CdS quantum dots could absorb best: 249 - $494 \mathrm{~nm}$. Similarly, the program outputted the wavelength ranges for the other cadmium chalcogenides and all other candidates. $\mathrm{CdSe}$, another prominent QD used in solar cells, had a size range of 1.2-4.1 $\mathrm{nm}$ [25] and the resultant wavelength range was 275-659 nm. Similarly, CdTe had its shortest and longest wavelengths as $274 \mathrm{~nm}$ and $784 \mathrm{~nm}$ respectively, resulting from having $1.2 \mathrm{~nm}$ as its smallest radius and $5.0 \mathrm{~nm}$ as its largest [26]. Our calculations gave similar results for the selected compounds from the III-V and IV-VI periodic groups. The IV-VI compounds, i.e. the lead chalcogenides, are considered as prominent materials for next-generation PV due to multiple factors, including their high dielectric constants and extinction constants that allow for easy separation of electrons and holes. Moreover, quantum dots composed from the III-V group compounds have band gaps from 0.7 to $6.2 \mathrm{eV}$, covering a wide range of spectra from ultraviolet (UV) to infrared region, thus exhibiting their crucial applications in PV [27]. By performing calculations on all our candidates, we were able to receive the following results, as displayed in Table 2 .

\section{TABLE 2}

Parameters utilized for ideal wavelength calculations using the mathematical model

\begin{tabular}{|l|l|l|l|l|}
\hline Materials & $\begin{array}{l}\text { Smallest } \\
\text { Radius / nm }\end{array}$ & $\begin{array}{l}\text { Largest } \\
\text { Radius / nm }\end{array}$ & $\begin{array}{l}\text { Shortest } \\
\text { Wavelength / nm }\end{array}$ & $\begin{array}{l}\text { Largest } \\
\text { Wavelength / nm }\end{array}$ \\
\hline $\mathrm{CdS}$ & 1.0 & 4.5 & 249 & 494 \\
\hline $\mathrm{CdSe}$ & 1.2 & 4.1 & 275 & 659 \\
\hline $\mathrm{CdTe}$ & 1.2 & 5.0 & 274 & 784 \\
\hline $\mathrm{PbS}$ & 2.4 & 7.3 & 651 & 2316 \\
\hline $\mathrm{PbSe}$ & 2.6 & 7.5 & 626 & 2658 \\
\hline $\mathrm{PbSe}$ & 1.4 & 7.0 & 718 & 3057 \\
\hline $\mathrm{PbTe}$ & 2.3 & 5.3 & 406 & 1005 \\
\hline $\mathrm{InAs}$ & 1.5 & 4.3 & 294 & 779 \\
\hline $\mathrm{InSb}$ & 4.5 & 5.7 & 761 & 1165 \\
\hline $\mathrm{GaAs}$ & 1.6 & 5.3 & 320 & 781 \\
\hline $\mathrm{GaP}$ & 2.2 & 4.5 & 435 & 537 \\
\hline $\mathrm{GaSb}$ & 2.5 & 4.0 & 582 & 914 \\
\hline $\mathrm{GaN}$ & 2.4 & 5.8 & 338 & 372 \\
\hline $\mathrm{ZnS}$ & 1.4 & 3.9 & 277 & 350 \\
\hline $\mathrm{AlAs}$ & 2.9 & 5.6 & 533 & 602 \\
\hline $\mathrm{AlN}$ & 2.8 & 7.8 & 206 & 230 \\
\hline
\end{tabular}

Based on the results, we can easily eliminate $\mathrm{GaP}$, $\mathrm{GaN}, \mathrm{ZnS}, \mathrm{AlAs}$, and AlN as there is very low tunability in their excitonic wavelengths based on their size. Furthermore, the low quality of these quantum dots' morphology only goes on to strengthen our decision of removing them. This lower quality of crystals compared to the cadmium and lead chalcogenides stands true for all III-V compounds, as shown by Chen et al. [23], further making them costlier to implement. This is due to the requirement of very fast nucleation and relatively slow growth processes in their synthesis. Our sole aim of considering the III-V compounds, despite their drawbacks, was to verify whether there is a span of wavelengths not absorbed by the II-VI and IV-VI group compounds that these quantum dots could absorb. A quick look at the results verifies that the $\mathrm{PbX}$ and $\mathrm{CdX}(\mathrm{X}=\mathrm{S}, \mathrm{Se}$, Te) QDs are at this point sufficient to cover whatever range the III-V QDs were covering. While it is true that three CdX QDs cover similar ranges, and so do the PbX QDs, we need to further analyze their properties before jumping to a conclusion. Thus, keeping the results for the III-V compounds and $\mathrm{ZnS}$ aside, we can display the remaining results on the AM 1.5 solar spectrum to better analyze the coverage of the solar spectrum, as shown in Figures 6a, b, and c.
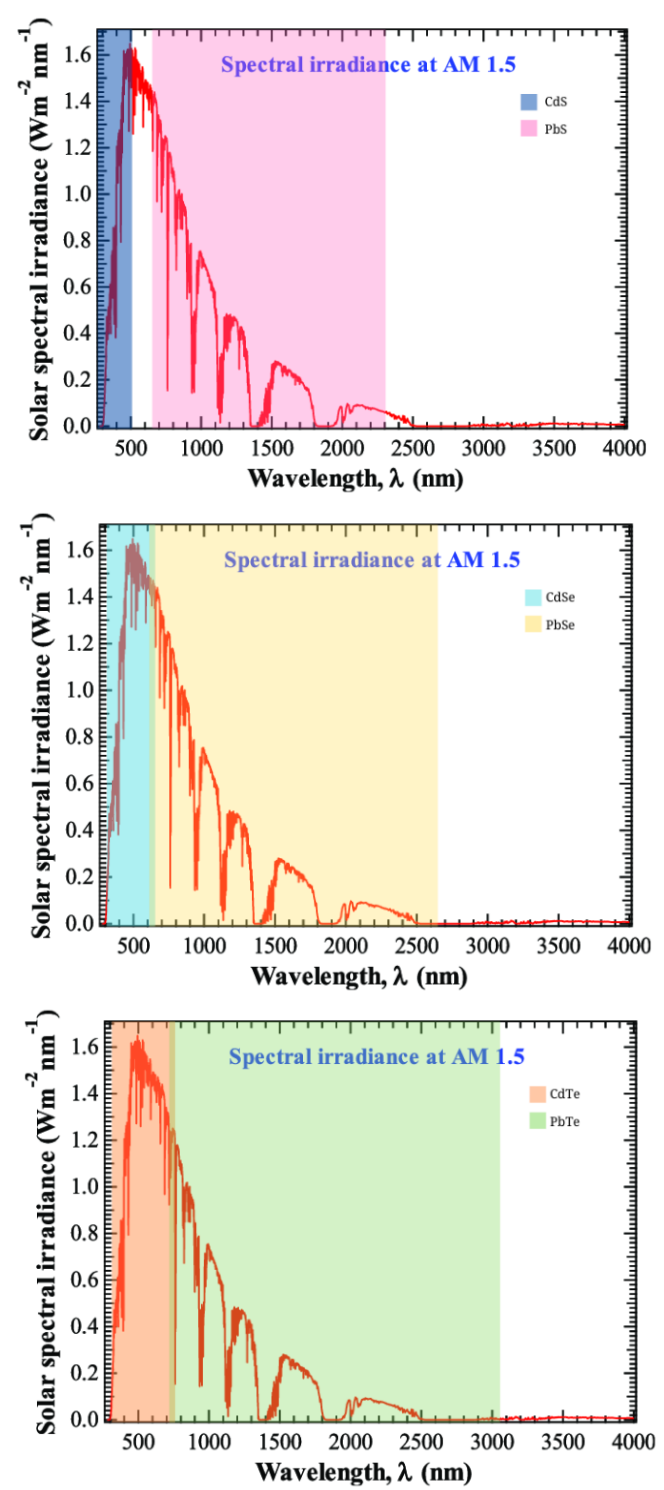

Fig -6: Top to bottom, the wavelength range of the solar spectrum covered by (a) $\mathrm{CdS}$ and $\mathrm{PbS}$, (b) CdSe and $\mathrm{PbSe}$, (c) CdTe and $\mathrm{PbTe}$ 
These figures do not represent the fact that these pairs of QDs will be used together. They have only been separated into three graphs for greater clarity about the range of each of our candidates. Thus, we can analyze the range covered by each of our shortlisted candidates. The absorbance range of each QD will also be slightly greater than that predicted by our model. This is because our longest wavelength for each material is the exact wavelength of light needed to excite a photon from the valence band to the conduction band in the largest QD of that material. However, this QD will also be able to absorb some photons with slightly shorter wavelengths, or slightly larger energies than the band gap energy of the QD, and the excess energy here is lost. While the selected materials cover most wavelengths without having to consider this, it should be noted that every material will also absorb in wavelengths slightly shorter than its range. Each QD with a specific size absorbs photons with energies greater than its band gap energy. While the wavelengths of these photons are majorly accounted for by QDs with smaller sizes, we consider the extra wavelengths only for the largest QD. Thus, for example, CdS will absorb light with wavelength lesser than $249 \mathrm{~nm}$ too, and this can be similarly understood for other materials.

The usage of the solar spectrum to display these wavelengths becomes essential because the vertical axis shows the solar spectral irradiance at each wavelength of light. The irradiance is highest in the visible light range, and we can then conclude that it is integral to employ QDs that absorb in this range. An initial look at the wavelength range spanned by $\mathrm{CdX}$ QDs as compared to PbX QDs would show that it is much shorter, and thus not as important. However, the fact that the CdX QDs absorb in the visible light range with a much higher spectral irradiance reflects that in a solar cell with a CdX QD and a PbX QD, the CdX QDs would contribute to about half of the output power if not more. Furthermore, since all three $\mathrm{CdX}$ and $\mathrm{PbX}$ QDs absorb in similar ranges respectively, it is clear that we will majorly need one material from both groups. This defines our methodology going forward as we select binary compounds from each of the two groups.

1) Cadmium Chalcogenides (CdX). All three of the cadmium chalcogenides cover parts of the visible light region. A quick look at the solar spectrum charts reflecting their wavelength ranges shows that $\mathrm{CdS}$ absorbs a much shorter range than $\mathrm{CdSe}$ and $\mathrm{CdTe}$. While $\mathrm{CdS}$ gives the advantage of absorbing an extra $20-30 \mathrm{~nm}$ in the UV range, the low spectral irradiance near the $250 \mathrm{~nm}$ mark shows that its incorporation will not yield many benefits as compared to the costs, discussed in greater detail ahead. The main focus remains on CdSe and CdTe. Between the two, there is not much difference in the shortest wavelength they can absorb, and even though CdTe absorbs slightly higher wavelengths, that range is already covered by the $\mathrm{PbX}$ QDs. Thus, the decision between the two must come on the basis of the quality of their respective quantum dots, which plays a considerable role in determining the durability and the effectiveness of the overall solar panel. Murray et al. had reported the synthesis of high-quality cadmium chalcogenides using dimethyl cadmium $\left(\mathrm{Cd}\left(\mathrm{CH}_{3}\right)_{2}\right)$ as the cadmium predecessor, and post this the synthesis of CdSe nanocrystals using this precursor had become well developed [28][29][30]. Yet, the synthesis of CdS and CdTe was not as advanced [31][32]. Dimethyl cadmium, however, is highly toxic, expensive, and unstable requiring very restricted equipment and making it an unviable option for large-scale synthesis. Peng at al. proposed the use of $\mathrm{CdO}$ as a precursor [33] which is a major step towards green chemistry and has low toxicity while being able to be used to synthesize high-quality nanocrystals with a controllable size for all three cadmium chalcogenides on a large scale. This method showed that the highest quantum efficiency amongst the three can be achieved for CdTe, with a value above $20 \%$. This would mean that CdTe can be used in QDSCs to collect the highest number of carriers as compared to the incident number of photons. Thus, CdTe is selected from the cadmium chalcogenide group and it is suggested that CdTe be synthesized using $\mathrm{CdO}$ as a precursor.

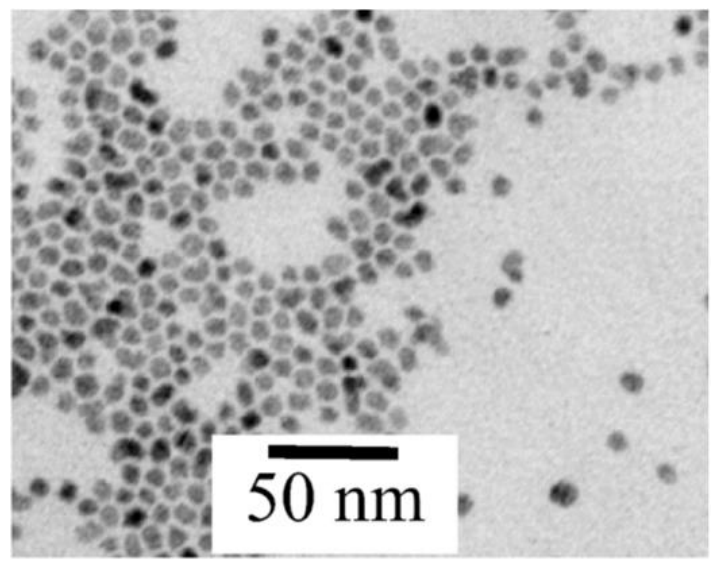

Fig -7: TEM pictures of CdTe nanocrystals synthesised by the $\mathrm{CdO}$ approach [32]

2) Lead Chalcogenides (PbX). Similar to Similar to the $\mathrm{CdX}$ QDs, the PbX QDs also cover similar ranges. However, they cover much larger ranges, and in the near-infrared region. Considering that $\mathrm{CdTe}$ absorbs till at least $784 \mathrm{~nm}$ and all $\mathrm{PbX}$ QDs start absorbing in wavelengths below that value, the starting point for the three can be considered the same. This doesn't mean that the fact that PbSe absorbs the shortest wavelengths (till $626 \mathrm{~nm}$ ) is ignored, as this could be helpful when it absorbs light of that wavelength that is not absorbed by CdTe. Furthermore, while PbTe absorbs the longest wavelength, the extremely low spectral irradiance at that length makes the benefits negligible. Thus, the selection will again have to be based on quality and synthesis. Contrasting to the many, well-documented reports on the synthesis of $\mathrm{PbS}$ and $\mathrm{PbSe} \mathrm{QDs}$, there has been very less research regarding the synthesis of PbTe QDs [34]. Thus, as their synthesis methods are not as developed and there is no proof of the ability to use them on a large-scale, $\mathrm{PbTe}$ will not be included. For both $\mathrm{PbSe}$ and $\mathrm{PbS}$, the material properties can be easily controlled using electron density flow design. They exhibit efficient utilization of low-intensity and low-energy photons and efficient collection of high-energy charges. Murray (2001) also suggested methods to synthesize colloidal $\mathrm{PbX}$ QDs, similar to the hot-injection method for CdX QDs. However, this method restricted control over the synthesis of smaller $\mathrm{PbX}$ QDs. Zhang et al. [35] have 
developed another effective non-hot injection-based synthesis method for $\mathrm{PbSe}$ and $\mathrm{PbS} \mathrm{QDs}$ that could be used to synthesise QDs in smaller sizes too while also simplifying the fabrication process for smaller sizes. This method could be used effectively, and it shows that solar cells with $\mathrm{PbS}$ QDs currently have $6.5 \%$ PCE while those with PbSe only have $2.65 \%$ PCE. While we know that their range of wavelengths absorbed is similar, this is a clear indication that $\mathrm{PbS}$ colloidal QDs are more suitable for use in QDSCs based on current developments in material and device characterizations. Thus, $\mathrm{PbS}$ is selected out of the $\mathrm{PbX}$ group of compounds.

Having considered the 16 materials, shortlisting to 6 materials based on wavelength ranges, and further narrowing down the search to 2 final materials after analyzing the quality and proven efficiencies of the QDs, the model can now be completed by analyzing the relative arrangement of both layers of QDs. It is interesting to note that after starting from 16 separate compounds, I finally arrived at 2 . It is best to lower the number as far as possible, because the greater the number of layers, the less light each subsequent less receives and the less power it contributes. Current developments relating to QDSCs with multiple films sandwiched together are limited, and thus incorporating a higher number of layers theoretically would reduce the viability of the model in the real world. Moreover, employing multiple layers also increases the cost of the solar panel while increasing extraction losses as it becomes tough to extract carriers from the layer in the bottom and to stop the electrons and holes to recombine. Contrastingly, multiple layers with different QD materials that absorb the same wavelength can be useful where the lower layer could absorb the same photon not absorbed by the upper layer due to the spacing between QDs. However, a cost-benefit analysis proves that this slight increase in the PCE will be overshadowed by the massive decrease in PCE with multiple films as per current developments. Hence, the goal was to select as few candidates as possible to cover most of the solar spectrum, and my analysis has revealed that this can be done with 2 materials. It now becomes integral to propose the relative arrangement of each of the selected QD materials in the solar panel and the suggested deposition methods.

\section{Deposition and Arrangement of $Q D s$}

For implementation in solar cells, a colloidal synthesis of QDs is preferred due to easy integration in the solar panel. With a solution-based synthesis method [36], colloidal QDs (CQDs) are ideal for our model as they can easily be synthesized and deposited on panels worldwide while being inexpensive to produce. The methods suggested above for the synthesis of CdTe and PbS QDs are used to produce CQDs. Furthermore, the size ranges stated above for the 16 materials, and thus CdTe and PbS, are also for CQDs. The use of CQDs in QDSCs looks very promising, as there have been regular and recent developments regarding producing high-quality CQDs with controllable size while also reducing their surface defects [23]. Thus, using colloidal CdTe and $\mathrm{PbS}$ QDs, we can deposit separate layers for each material. Currently, most high-efficiency CQD PVs use a thin-film solar cell structure, and thus our model would involve using two films together as we have two separate QD materials. It is then important to decide on the deposition method for each layer. While there are many surface-related issues regarding the employment of CQDs, there are brief solutions provided to counter these problems.

The formation of a conductive CQD thin film involves the usage of a layer-by-layer process [LBL], where the deposition of the CQD film is repeatedly performed. This process may produce cracks on the surface, but these cracks can be reduced by the ligand exchange process. This involves replacing the long organic ligands on the non-stoichiometric surface with mercaptopropionic acid ligands containing sulfur (anion), as proposed by Ip et al [37]. However, LBL is a time-consuming process and the electronic properties of the CQDs are modified by the environment during the ligand exchange process. Hence, the reproducibility CQD PVs is reduced, making them unsuitable for commercialization. However, this can be solved by modifying the surface with ammonium iodide $\left(\left(\mathrm{NH}_{4}\right) \mathrm{I}\right)$ [38].

During the formation of the films, the order of the materials must be considered too. A tandem solar cell structure can be utilized for effectively incorporating two materials. It is known that photons with energy lower than the band gap energy of the QD do not contribute to the power generated as they are not absorbed. Moreover, each photon with an energy greater than the band gap energy of the QD contributes one electron to the current, while the energy exceeding the band gap is lost. The knowledge of these concepts can be used to efficiently arrange the two materials. In a tandem structure, the films fabricated for each material are stacked. The photon must first strike the film containing the material with the higher band gap energy because then light with higher energy will be absorbed with a higher output voltage. This material will also be transparent for low energy light which can be passed on to the second absorber film with the lower band gap. Thus, as the band gap is larger for sizes of CdTe QDs that I have incorporated, the CdTe film must receive light from the sun first, followed by the $\mathrm{PbS}$ film. The LBL process must then be used to first deposit a film of $\mathrm{PbS}$ CQDs followed by a film of CdTe CQDs. This arrangement would hence allow us to harness a higher PCE as both absorbers would receive light and maximum photons will contribute to the current in the solar cell.

Another topic that must be addressed is that of the size of the CQDs deposited. The size range suggested earlier and used for the mathematical analysis was based on the current range of CQDs that can be synthesized for commercial use. CQD solutions that are created involve such size ranges. During the synthesis process, the radius of the QDs must be evenly spread over the range so that light can be absorbed throughout the range of wavelengths. However, each section of the film must also have QDs across the range of radii, for eg. $1.2-5.0 \mathrm{~nm}$ for CdTe. These two requirements become difficult to incorporate together, considering that chemical properties of CQDs also restrict their maximum concentration in solutions. However, manufacturers must try to achieve both requirements to the greatest extent possible. This is 
essential to ensure that apart from an extensive range of the solar spectrum being absorbed, the model even produces high power per unit area. As developments lead to higher control of the sizes of the CQDs, each pass during the LBL process can involve CQDs of different sizes to evenly cover each section of the film with CQDs of all sizes available. Here, it must be ensured that CQDs with the smallest sizes are deposited on the top in the respective film as they would have the largest band gap energies.

The number of CQDs incorporated in the solar panel is directly linked to the concentration of the solution, and higher concentrations can allow for a greater PCE. However, higher concentrations also create problems with respect to surface ligands and can cause defects. Zhao et al. conducted research to determine the critical concentration that allows for the greatest PCE [39], and these considerations can be employed in our model to determine the ideal concentration for CQD solutions of $\mathrm{CdTe}$ and $\mathrm{PbS}$. With this, we can arrive at a finalized model for a highly efficient QDSC. While the model will still have significant energy losses, as will be discussed further, the structure which is developed and shown in Figure 8 can be used as a stepping-stone to producing low-cost and efficient QDSCs.

Highly Efficient QDSC Model

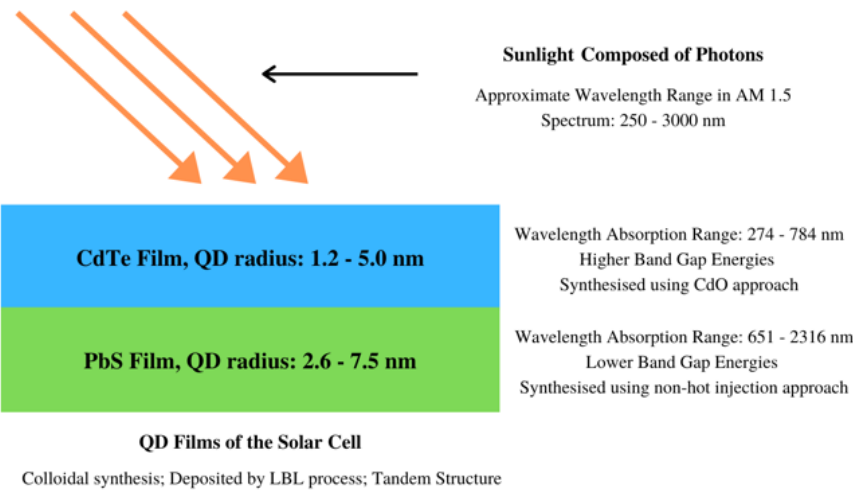

Fig. 8 Finalised QD film structure of our QDSC model for high PCE based on optical properties of constituent QD materials and synthesis and deposition considerations

\section{Energy Losses in the QDSC Model}

Having selected the materials, arrangement, and synthesis and deposition methods for the QDSC, it is also important to evaluate the energy losses in the solar panel. While there are multiple avenues for energy losses, my analysis will be focused on the specific ones arising from the incorporation of QDs. In analysing the energy losses in solar cells employing CQDs, the work of Song et al. [23], also having been cited earlier, was of great significance. A major source of energy losses in CQD PVs is electron recombination, where the electron recombines with the hole in the QD before contributing to the current. It must be noted that I am not talking about the recombination that takes place after the electron has moved around the entire circuit. This problem is present for bulk material PVs too, but the loss in energy is not as significant due to better device characterizations than QDSCs. While PbS has a high dielectric constant and would hence face lesser troubles regarding electron recombination, this problem would be more significant with CdTe. Even if my model works in an ideal manner and maximum photons are converted to excited electrons, the recombination of the electron would mean that there is no effective increase in the PCE. Hence, an effective solution to this issue is the usage of an electron blocking layer (EBL). This can be employed at the interface between the CQD films and the metal electrode. It works by blocking the hole transfer at the interface, thus preventing recombination. Advantageously, the EBL would be placed between the top layer, i.e. CdTe, and the metal electrode, as the major worry concerning electron recombination was $\mathrm{CdTe}$.

The surface characterisations in solar cells involving CQDs are also a major hindrance to a higher PCE. The high surface-to-area ratio of CQDs is the cause of many surface defects, and the use of surface ligands can reduce these defects. Additional passivation of CQDs using halide atoms works to reduce the surface defects while increasing the photoluminescence and quantum yield of the CQDs [40], as shown in Figure 9a and b. Furthermore, this prevents the oxidation of CQDs, improving their air stability [41]. While the surface defects cause energy losses due to acting as an impediment to effective absorption of protons and extraction of electrons, the usage of halide atoms effectively reduces these energy losses.

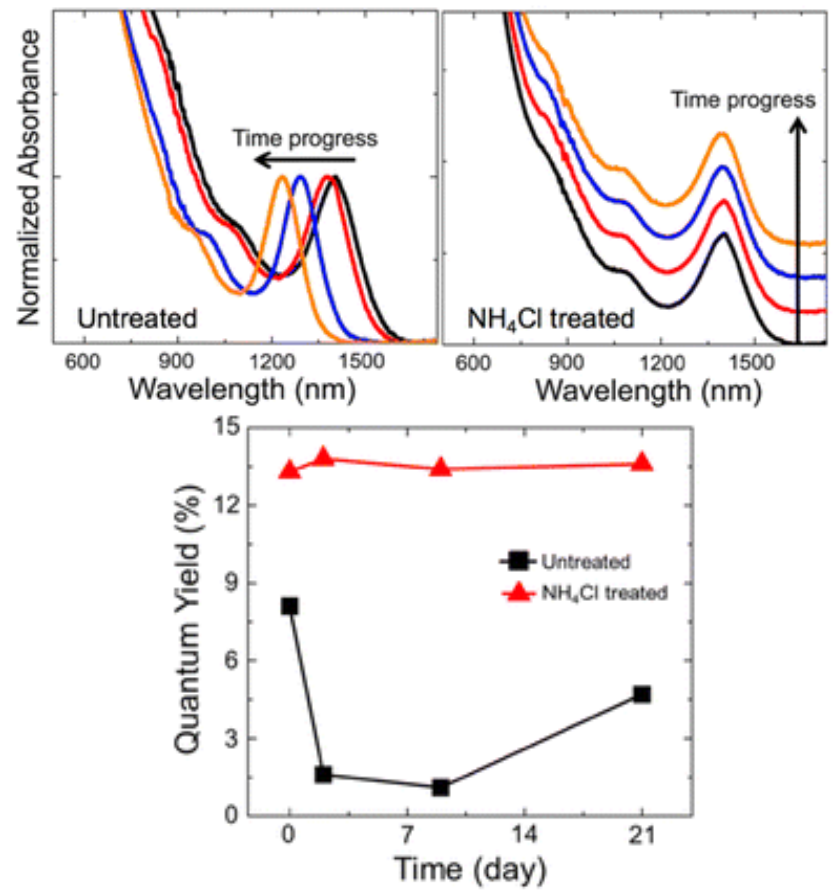

Fig -9: Top to bottom, the graphs display (a) the increased absorbance and thus photoluminescence and (b) the increased quantum yield of CQDs postpassivation using halide atoms [40]

Finally, another issue with QDSCs arises where the increase in the energy band gap and discrete energy level due to quantum confinement causes a decrease in the density of state. This reduces the amount of light absorbed around the energy band gap, causing the external quantum efficiency (EQE) spectrum for CQD PVs to show a significant decrease in efficiency near the energy band gap. While this is a unique 
characteristic of CQDs, it can be solved using plasmons of metal nanoparticles to shift the spectrum of the incident sunlight to the region in which CQDs can absorb large amounts of light [42]. This does not affect the absorption of our selected QDs and the spectral irradiance for the solar spectrum, but only works to shift the EQE spectrum. Hence, an energy loss resulting from a drop in the efficiency in this spectrum can be avoided.

With this, the major sources of energy losses in the case of CQD PVs and especially concerning the CQDs have been analysed and solutions have been proposed to reduce these losses. These solutions may not be immediately implementable at a commercial level, but their success on an experimental level verifies that the future holds prospects of minimising the major energy losses in QDSCs. Overall, post modelling a QD film section of a solar cell based on absorbing more photons that contribute electrons to the current, several solutions have been provided to the problems that may arise with this method. It can thus be said that an effective framework has been developed to increase the PCE of QDSCs.

\section{DISCUSSIONS AND FURTHER DIRECTIONS}

The model developed is one that can be used to manufacture higher efficiency QDSCs without relying on future developments. At every step of the way, I ensured that the methods suggested and the propositions put forward were ones that could be implemented on a commercial scale. Globally, there have been numerous developments in the run for creating extremely efficient and advanced quantum dot solar cells. Scientists at the University of Queensland, Australia have set a new world record for the highest efficiency QDSC, creating a perovskite material based solar cell with $16.6 \%$ efficiency [43]. However, they were able to achieve this feat only by creating a $0.1 \mathrm{~cm} 2$ panel. While the PCE value is impressive, it is integral to understand that such a small size has no use in the commercial world. In the future, such QDSCs may be used widely post years of research and development, providing extremely high efficiencies and making solar energy cost-competitive with fossil fuels. However, they do not satisfy the requirement of the present. The methodology I used was designed to ensure that the model we propose is one that could be implemented commercially very soon. By selecting sizes for QDs that are easily fabricated, incorporating quality considerations during shortlisting materials, using the easily synthesized CQDs, and noting the cost of all methods and processes used while also trying to maximise efficiency, the framework created is one that is built for possible use in the commercial world.

However, there are numerous limitations associated with the model that cannot be ignored. Firstly, despite certain efficiency limitations with tandem structures, they have been incorporated because it is otherwise not possible to use multiple layers of materials apart from multi-junction QDSCs, which have not shown considerable success on a large scale. Secondly, it is crucial to understand that the model only discusses the incorporation of quantum dots in the solar cell. Every QDSC has multiple other components apart from the QDs itself. This paper only focused on QDs and methods related to increasing their effectiveness in the solar cell. It is beyond the scope of this paper to discuss methods to increase the efficiency of every single component of the QDSC. Thirdly, by not integrating a detailed consideration of the interaction of the QD films with the other components, the processes that increase the efficiency of the QDs may decrease the efficiency of those components. For the QDs, the main focus was on the absorption ranges and determining these through the use of available size data. Due to the high integration of multiple subjects in QDSCs, it was sensible to look at the model from a material science or electronic engineering perspective. Thus, synthesis and deposition methods were considered, along with tackling electron recombination related problems. All in all, the paper tries to improve the usage of QDs in QDSCs to deliver a greater PCE while trying to consider as much of their chemical and electrical problems as possible. There may also be optical concepts that have been overlooked, such as refraction in the QD films and back reflection. However, the aim was never to give an all-encompassing model. This model is only one that can be used by specialists across these fields to fine-tune the other sections and concepts to thus deliver a complete, high-efficiency QDSC.

With increasing development relating to third-generation solar cells and sustainable solar cells, there are many prospects of altering the model. I had chosen CdTe and PbS despite being aware of their toxicity because currently there are no non-toxic alternatives that come close. However, research into green QDs ensures that this methodology could again be used with only considering these QDs. While the CdTe synthesis method considered falls under green chemistry, a larger part of our paper fails to incorporate green chemistry due to its limited development. Moreover, concepts like multiple exciton generation have been discussed earlier in the paper. MEG can drastically increase the PCE of QDSCs, and while it has recently been demonstrated in a functioning QDSC [44], it had not been considered due to very few successful experiments that incorporate it. Postdevelopment of methods to easily use MEG on a large scale, with intermediate band PVs being the leading choice, materials and structures must be selected based on their capability to generate multiple excitons from one photon. Similarly, increasing interest in heterojunction solar cells and perovskite material based solar cells shows that they could also be incorporated into my research. Thus, the model can be altered drastically to incorporate future developments. Today, as stated earlier, it stands as a basic framework for material scientists and engineers to use and build upon with more detailed considerations of each component to create QDSCs that have the potential to make solar energy the primary source of energy worldwide.

\section{CONCLUSIONS}

QDSCs have been attracting much attention because QDs can be tuned to absorb a wide range of wavelengths of light. The mathematical model used was successful in outputting the range absorbed by each material considering its size. Based on this and material characterizations, $\mathrm{CdTe}$ and $\mathrm{PbS}$ 
were shortlisted which absorb $274-784 \mathrm{~nm}$ and $651-2316$ $\mathrm{nm}$ of lights respectively. By using colloidal solutions of both QDs and depositing them using the LBL process, we were able to create a tandem structure for QDs in QDSCs where CdTe QDs were placed above PbS QDs due to having higher band gaps. Finally, methods like employing an electron blocking layer and passivating the surface with halide atoms were proposed to minimise energy losses. While the final model has certain limitations, it is a basic yet effective structure that can be employed in QDSCs while considering the other components to deliver a high PCE.

\section{ACKNOWLEDGMENT}

For guiding me throughout my work, I would like to acknowledge Dr. Maarij Syed, Professor of Physics and Optical Engineering, Rose-Hulman Institute of Technology, Terra Haute, US. Through multiple online sessions, Prof. Syed helped me to achieve my goals of creating a model for a higher efficiency quantum dot solar cell. It was under his tutelage that I could conduct undergraduate level research, and I am grateful to him for helping me unravel the complexities of the optical physics behind quantum dots. His motivation has allowed me to become deeply interested in the field, pushing me to conduct further research.

\section{REFERENCES}

[1] Ren21.net. (2020). Retrieved 12 July 2020, from https://www.ren21.net/wpcontent/uploads/2019/05/gsr_2019_full_report_en.pdf.

[2] Aggarwal, V. (2020). Solar Panel Efficiency: What Panels Are Most Efficient? | EnergySage. Solar News. Retrieved 12 July 2020, from https://news.energysage.com/what-are-the-most-efficient-solar-panelson-the-market/.

[3] Semonin, O., Luther, J., \& Beard, M. (2012). Quantum dots for nextgeneration photovoltaics. Materials Today, 15(11), 508-515. https://doi.org/10.1016/s1369-7021(12)70220-1

[4] Miller, E.Y.O.D, CLEO: Science and Innovation (2012), paper CF2J.1

[5] Green, M. (2003). Third Generation Photovoltaics. Materials Today, 6(11), 51. https://doi.org/10.1016/s1369-7021(03)01133-7

[6] Conibeer, G. (2007). Third-generation photovoltaics. Materials Today, 10(11), 42-50. https://doi.org/10.1016/s1369-7021(07)70278-x

[7] Aroutiounian, V., Petrosyan, S., Khachatryan, A., \& Touryan, K (2001). Quantum dot solar cells. Journal Of Applied Physics, 89(4), 2268-2271. https://doi.org/10.1063/1.1339210

[8] Ocf.berkeley.edu. (2020). Retrieved 12 July 2020, from https://www.ocf.berkeley.edu/ jmlvll/labreports/quantumDots/quantumDots.pdf.

[9] Cdn.intechopen.com. (2020). Retrieved 12 July 2020, from https://cdn.intechopen.com/pdfs/47671.pdf

[10] magazine, pv. (2020). Quantum dots: The pros and cons in PV. pv magazine International. Retrieved 12 July 2020, from https://www.pvmagazine.com/magazine-archive/quantum-dots-the-pros-and-cons-inpv_100010173/.

[11] Gueymard, C., Myers, D., \& Emery, K. (2002). Proposed reference irradiance spectra for solar energy systems testing. Solar Energy, 73(6), 443-467. https://doi.org/10.1016/s0038-092x(03)00005-7

[12] Physics.unm.edu. (2020). Retrieved 12 July 2020, from http://physics.unm.edu/Courses/Fields/Phys491/Notes/TISEInfiniteSqu are.pdf.

[13] Brus, L. (1983). A simple model for the ionization potential, electron affinity, and aqueous redox potentials of small semiconductor crystallites. The Journal Of Chemical Physics, 79(11), 5566-5571. https://doi.org/10.1063/1.445676

[14] Zhao, Q., Hazarika, A., Chen, X., Harvey, S., Larson, B., \& Teeter, G. et al. (2020). High efficiency perovskite quantum dot solar cells with charge separating heterostructure. Retrieved 12 July 2020, from https://www.nature.com/articles/s41467-019-10856-Z
[15] Ouantum Dots Nanoparticles \& Ouantum Dots | Knowledge Base Nanomaterials. Nanopartikel.info. (2020). Retrieved 12 July 2020, from https://www.nanopartikel.info/en/nanoinfo/materials/quantumdots/material-information

[16] Paschotta, D. (2020). Band Gap. Rp-photonics.com. Retrieved 12 July 2020, from https://www.rp-photonics.com/band_gap.html.

[17] Pellegrini, G., Mattei, G., \& Mazzoldi, P. (2005). Finite depth square well model: Applicability and limitations. Journal Of Applied Physics, 97(7), 073706. https://doi.org/10.1063/1.1868875

[18] Trindade, T., O'Brien, P., \& Pickett, N. (2001). Nanocrystalline Semiconductors: Synthesis, Properties, and Perspectives. Chemistry Of Materials, 13(11), 3843-3858. https://doi.org/10.1021/cm000843p

19] NSM Archive - Physical Properties of Semiconductors. Ioffe.ru. (2020). $\begin{array}{llll}\text { Retrieved } & 12 & \text { July } & \text { from }\end{array}$ http://www.ioffe.ru/SVA/NSM/Semicond/.

[20] Hal.archives-ouvertes.fr. (2020). Retrieved 12 July 2020, from https://hal.archives-ouvertes.fr/jpa-00213615/document.

[21] Ecse.rpi.edu. (2020). Retrieved 12 July 2020, from https://www.ecse.rpi.edu/ schubert/Educational-resources/MaterialsSemiconductors-III-V-arsenides.pdf.

[22] Ecse.rpi.edu. (2020). Retrieved 12 July 2020, from https://www.ecse.rpi.edu/ schubert/Educational-resources/MaterialsSemiconductors-III-V-nitrides.pdf.

[23] Song, J., \& Jeong, S. (2017). Colloidal quantum dot based solar cells: from materials to devices. Nano Convergence, 4(1). https://doi.org/10.1186/s40580-017-0115-0

[24] Veerathangam, K., Pandian, M., \& Ramasamy, P. (2018). Sizedependent photovoltaic performance of cadmium sulfide (CdS) quantum dots for solar cell applications. Journal Of Alloys And Compounds, 735 ,

202-208 https://doi.org/10.1016/j.jallcom.2017.11.055

[25] Kashyout, A., Soliman, H., Fathy, M., Gomaa, E., \& Zidan, A. (2012) CdSe Quantum Dots for Solar Cell Devices. International Journal Of Photoenergy, 2012, 1-7. https://doi.org/10.1155/2012/952610

[26] Mezrag, F., \& Bouarissa, N. (2019). Pseudopotential Study of CdTe Quantum Dots: Electronic and Optical Properties. Materials Research 22(3). https://doi.org/10.1590/1980-5373-mr-2017-1146

27] Chen, Z., Sun, C., Guo, W., \& Chen, Z. (2020). Colloidal III-V Nitride Quantum Dots. Retrieved 12 July 2020, from http://dx.doi.org/10.5772/intechopen.70844.

[28] Peng, X., Manna, L., Yang, W., Wickham, J., Scher, E., Kadavanich, A., \& Alivisatos, A. (2000). Shape control of CdSe nanocrystals. Nature, 404(6773), 59-61. https://doi.org/10.1038/35003535

[29] Chu, H., Li, X., Chen, G., Zhou, W., Zhang, Y., \& Jin, Z. et al. (2005) Shape-Controlled Synthesis of CdS Nanocrystals in Mixed Solvents. Crystal Growth \& Design, 5(5), 1801-1806. https://doi.org/10.1021/cg050068w

[30] Peng, X., Wickham, J., \& Alivisatos, A. (1998). Kinetics of II-VI and III-V Colloidal Semiconductor Nanocrystal Growth: "Focusing" of Size Distributions. Journal Of The American Chemical Society, 120(21), 5343-5344. https://doi.org/10.1021/ja9805425

[31] Vossmeyer, T., Katsikas, L., Giersig, M., Popovic, I., Diesner, K., \& Chemseddine, A. et al. (1994). CdS Nanoclusters: Synthesis, Characterization, Size Dependent Oscillator Strength, Temperature Shift of the Excitonic Transition Energy, and Reversible Absorbance Shift. The Journal of Physical Chemistry, 98(31), 7665-7673. https://doi.org/10.1021/j100082a044

[32] Mikulee, F.; Ph.D. Thesis, MIT, Boston, 1998.

[33] Peng, Z., \& Peng, X. (2001). Formation of High-Quality CdTe, CdSe, and $\mathrm{CdS}$ Nanocrystals Using $\mathrm{CdO}$ as Precursor. Journal Of The American Chemical Society, 123(1), 183-184. https://doi.org/10.1021/ja003633m

[34] Murphy, J., Beard, M., Norman, A., Ahrenkiel, S., Johnson, J., \& Yu, P. et al. (2006). PbTe Colloidal Nanocrystals: Synthesis, Characterization, and Multiple Exciton Generation. Journal Of The American Chemical Society, 128(10), 3241-3247. https://doi.org/10.1021/ja0574973

35] Zhang, J., Gao, J., Miller, E., Luther, J., \& Beard, M. (2013). Diffusion-Controlled Synthesis of PbS and PbSe Quantum Dots with in Situ Halide Passivation for Quantum Dot Solar Cells. ACS Nano, 8(1), 614-622. https://doi.org/10.1021/nn405236k

[36] Hines, M., \& Scholes, G. (2003). Colloidal PbS Nanocrystals with Size-Tunable Near-Infrared Emission: Observation of Post-Synthesis Self-Narrowing of the Particle Size Distribution. Advanced Materials, 15(21), 1844-1849. https://doi.org/10.1002/adma.200305395 
[37] Ip, A., Thon, S., Hoogland, S., Voznyy, O., Zhitomirsky, D., \& Debnath, R. et al. (2012). Hybrid passivated colloidal quantum dot solids. Nature Nanotechnology, 7(9), 577-582. https://doi.org/10.1038/nnano.2012.127

[38] Kim, S., Noh, J., Choi, H., Ha, H., Song, J., \& Shim, H. et al. (2014) One-Step Deposition of Photovoltaic Layers Using Iodide Terminated PbS Quantum Dots. The Journal Of Physical Chemistry Letters, 5(22), 4002-4007. https://doi.org/10.1021/jz502092x

[39] Zhang, T., Zhao, H., Riabinina, D., Chaker, M., \& Ma, D. (2010). Concentration-Dependent Photoinduced Photoluminescence Enhancement in Colloidal PbS Quantum Dot Solution. The Journal Of Physical Chemistry C, 114(22), 10153-10159. https://doi.org/10.1021/jp1025152

[40] Woo, J., Ko, J., Song, J., Kim, K., Choi, H., \& Kim, Y. et al. (2014). Ultrastable PbSe Nanocrystal Quantum Dots via in Situ Formation of Atomically Thin Halide Adlayers on $\mathrm{PbSe}(100)$. Journal of The American Chemical Society, 136(25), 8883-8886. https://doi.org/10.1021/ja503957r

[41] Bae, W., Joo, J., Padilha, L., Won, J., Lee, D., \& Lin, Q. et al. (2012). Highly Effective Surface Passivation of PbSe Quantum Dots through Reaction with Molecular Chlorine. Journal Of The American Chemical Society, 134(49), 20160-20168. https://doi.org/10.1021/ja309783v

[42] Baek, S., Song, J., Choi, W., Song, H., Jeong, S., \& Lee, J. (2015). A Resonance-Shifting Hybrid n-Type Layer for Boosting Near-Infrared Response in Highly Efficient Colloidal Quantum Dots Solar Cells. Advanced $\quad$ Materials, 27(48), 8102-8108. https://doi.org/10.1002/adma.201503642

[43] Hutchins, M. (2020). A quantum dot solar cell with $16.6 \%$ efficiency. pv magazine International. Retrieved 12 July 2020, from https://www.pv-magazine.com/2020/02/19/a-quantum-dot-solar-cellwith-16-6-efficiency/.

[44] Semonin, O., Luther, J., Choi, S., Chen, H., Gao, J., Nozik, A., \& Beard, M. (2011). Peak External Photocurrent Quantum Efficiency Exceeding 100\% via MEG in a Quantum Dot Solar Cell. Science, 334(6062), 1530-1533. https://doi.org/10.1126/science.1209845 